\title{
A targeting peptide improves adenovirus-mediated transduction of a glioblastoma cell line
}

\author{
DONGYANG WANG ${ }^{1}$, WENBO LI ${ }^{1}$, HANG ZHANG $^{1}$, QINWEN MAO $^{2}$ and HAIBIN XIA ${ }^{1}$ \\ ${ }^{1}$ Laboratory of Gene Therapy, Department of Biochemistry, College of Life Sciences, \\ Shaanxi Normal University, Xi'an, Shaanxi 710062, P.R. China; ${ }^{2}$ Department of Pathology, \\ Northwestern University, Feinberg School of Medicine, Chicago, IL 60611, USA
}

Received December 31, 2013; Accepted February 12, 2014

DOI: $10.3892 /$ or.2014.3065

\begin{abstract}
The progress of the application of adenovirus in cancer gene therapy is hindered by the lack of expression of native adenovirus receptor on a variety of cancer types. Hence, strategies are needed to retarget the adenoviral vector to non-native cellular surface receptors. In the present study, a new peptide SWDIAWPPLKVP, capable of selectively targeting a human glioblastoma cell line A172, was identified by direct biopanning of phage-display peptide libraries. The binding activity of the phage displaying SWDIAWPPLKVP peptide in A172 was more than 10-fold higher than that of the control phage. We then inserted the selected peptide SWDIAWPPLKVP into adenoviral hexon protein, and observed that the modified Ad5 had increased infectivity in A172 cells, compared with that in control cell lines. These findings demonstrated that a peptide acquired through phage display can mediate cell-specific Ad retargeting when inserted into Ad hexon, suggesting an approach for targeting adenoviral infection to specific cancer cells.
\end{abstract}

\section{Introduction}

Adenovirus (Ad) has shown promise in cancer therapy. Ad has a high viral titer and high transduction efficiency for both proliferating and quiescent cells; it permits the insertion of large fragments of foreign DNA, and the viral DNA does not integrate into the host (1). These characteristics make it an ideal choice compared to other vectors. However, the progress of the application of Ad in cancer gene therapy is hindered by the lack of expression of native adenovirus receptor on a variety of cancer types (2-4). As such, strategies are needed to retarget the Ad vector to non-native cellular surface receptors.

Correspondence to: Professor Haibin Xia, Laboratory of Gene Therapy, Department of Biochemistry, College of Life Sciences, Shaanxi Normal University, 199 South Chang'an Road, Xi'an, Shaanxi 710062, P.R. China

E-mail: hbxia2001@163.com

Key words: targeting, phage display, adenovirus, human glioblastoma
Most of the commonly used adenoviral vectors are based on the serotype 5 (Ad5). Host cell entry of Ad5 is a two-step process with two major interactions between Ad coat proteins and cell surface receptors. First, high-affinity interaction occurs when the fiber knob domain binds to its cognate primary receptor, the coxsackie and Ad receptor (CAR) (5-8). Second, cellular integrins interact with Arg-Gly-Asp (RGD) motifs in the penton base to promote the viral internalization $(9,10)$.

Strategies have been utilized to transductionally target Ad-based vectors, which include adapter-based approach $(11,12)$, creation of fiber-chimeric Ad (13-16) and direct incorporation of peptides into viral capsid (17-21). The latter has been preferred for cell-specific Ad retargeting. Fiber proteins (either $\mathrm{C}$ terminus or HI-loop) and hexon are commonly used capsid proteins for the incorporation of targeting ligands.

In the present study, a new peptide capable of selectively targeting a human glioblastoma cell line A172 was identified by direct biopanning of phage-display peptide libraries. The peptide was then utilized to modify adenoviral hexon, which was shown to be able to mediate specific adenoviral binding to A172 glioblastoma cells and improve the adenoviral transduction efficiency.

\section{Materials and methods}

Cells and phage libraries. The human glioblastoma cell lines A172, U87 and human glioma cell line U251 were purchased from the Cell Bank of the Chinese Academy of Sciences (Shanghai, China); the human embryonic kidney cell line HEK 293 and lung carcinoma cell line A549 were obtained from the American Type Culture Collection (ATCC; Manassas, VA, USA). All the cells were grown in high glucose DMEM (Gibco, Carlsbad, CA, USA) containing 10\% (v/v) newborn calf serum with $100 \mathrm{U} / \mathrm{ml}$ penicillin and $0.1 \mathrm{mg} / \mathrm{ml}$ streptomycin. PhD-12 library displaying 12 random amino acids were obtained from New England Biolabs (Ipswich, MA, USA).

In vitro selections. BRASIL protocol (22) was optimized and used for our phage selections. In the subtraction step, HEK 293 cells were detached and collected with $5 \mathrm{mM}$ EDTA in PBS, then washed with serum-free DMEM, and re-suspended in $300 \mu \mathrm{l}$ DMEM containing $1 \%$ BSA with $5 \times 10^{6}$ cells and incu- 
bated with $2 \times 10^{10}$ pfu phage within $1.5 \mathrm{ml}$ Eppendorf tubes at $4^{\circ} \mathrm{C}$ for $3 \mathrm{~h}$ with slow shaking. Then, $300 \mu \mathrm{l}$ of the cellphage suspension was centrifuged at $500 \mathrm{x}$ g for $10 \mathrm{~min}$ and the supernatant was incubated with $50 \mu \mathrm{l}$ RPMI-1640 medium containing $1 \%$ BSA with $5 \times 10^{6}$ A 172 cells for $3 \mathrm{~h}$ at $4^{\circ} \mathrm{C}$. Then, the $350 \mu \mathrm{l}$ cell-phage suspension was gently transferred to the top of $800 \mu \mathrm{l}$ non-miscible organic lower phase [dibutyl phthalate: cyclohexane $=9: 1(\mathrm{v}: \mathrm{v})]$ in $1.5 \mathrm{ml}$ Eppendorf tube and centrifuged at $10,000 \mathrm{x}$ for $10 \mathrm{~min}$ at $4^{\circ} \mathrm{C}$. The tube was snap frozen in liquid nitrogen and the cell-phage pellet at the bottom of the tube was sliced off and transferred to a new tube incubated with the host E. coli strain ER2738 to be recovered, amplified and tittered following the Ph.D. Phage Display Libraries instruction manual from New England Biolabs. The entire subtraction/selection cycle was repeated four times.

Immunofluorescence staining. A172 cells were placed in 6-well plates at the density of $2 \times 10^{5}$ cells/well. Twenty-four hours later, culture medium was removed and the cells were rinsed with PBS twice. Then $2 \times 10^{11}$ pfu phages diluted in blocking buffer [3\% (m/v) BSA in serum-free DMEM] were added into every well and incubated with the cells for 90 min at $4^{\circ} \mathrm{C}$. After the incubation, cells were washed with PBST five times and then fixed by $4 \%$ paraformaldehyde at room temperature for $20 \mathrm{~min}$. Then, the cells were rinsed three times in PBS for 5 min each. The antibody against M13 phage was used as the primary antibody followed by the SABC-Cy3 solution. DAPI was used for nuclear counter staining.

Determination of phage binding efficiency. Phages of $5 \times 10^{9}$ pfu were incubated with $1 \times 10^{6}$ cells at $4^{\circ} \mathrm{C}$. After elution and titration, the phage binding efficiency was calculated as number of output phage/number of input phage.

Specific binding of the phages and cells by ELISA. A172, U87, A549 and U251 cells (1×10 cells) were plated on 96-well plates, respectively. Twenty-four hours later, cells were fixed with $4 \%$ formaldehyde/PBS for $15 \mathrm{~min}$ and washed three times with PBS. Before cells were incubated with phages for $1.5 \mathrm{~h}$ at $4^{\circ} \mathrm{C}$, cells were first blocked with PBS containing $1 \%$ BSA for $30 \mathrm{~min}$ at room temperature to avoid non-specific binding of anti-M13 antibody. Then, the phage bound to cells was quantified following the normal direct ELISA protocol using mouse monoclonal antibody to M13 Bacteriophage Coat Protein g8p (Abcam, Cambridge, MA, USA) as primary antibody, horseradish peroxidase conjugated-goat anti-mouse IgG antibody (ZSGB-Bio, Beijing, China) as secondary antibody, ABTS (Shanghai Sangon Biotechnology, Shanghai, China) as the substrate, and a Multiskan MK3 microplate reader (Thermo Fisher Scientific, Waltham, MA, USA) for visualization at $405 \mathrm{~nm}$.

Construction and propagation of adenoviral vector. The recombinant replication deficient Ad5 vector used in the present study contains an eGFP expression cassette driven by human cytomegalovirus intermediate-early promoter in E1 region. The peptide SWDI selected from phage library was inserted into the HVR5 of hexon protein in the Ad5 capsid by one step ligation as previously described (21). The nucleotide sequence encoding SWDI peptide (SWDIAWPPLKVP), flanked by $B a m \mathrm{HI}$ and $S f u \mathrm{I}$, was ligated with BamHI and SfuI digested
Table I. Sequences displayed in individual phage clones.

\begin{tabular}{llc}
\hline Phage clone & Consensus sequences & Frequency \\
\hline $12-2,12-4,12-5$, & SWDIAWPPLKVP & 6 \\
$12-9,12-11,12-12$ & & \\
$12-1,12-6,12-13$ & NILPYNKTMLVK & 3 \\
$12-3$ & SSSNTTTKHVFT & 1 \\
$12-7$ & DSYYTKTERNTH & 1 \\
$12-8$ & YEFPRSWDMETN & 1 \\
$12-10$ & NRPDSAQFWLHH & 1 \\
$12-14$ & GWEVTWPASYAF & 1 \\
\hline
\end{tabular}

pRGHMAd5. The obtained plasmid, termed pRGHMAd5SWDI, was then confirmed by enzyme digestion and DNA sequencing. The recombinant vector was linearized by $\mathrm{PacI}$ and then transfected into HEK 293 to package the modified Adenoviral vector Ad5 Hexon-SWDI. The adenovirus was further propagated in HEK 293 cells and was purified by cesium chloride gradient methods. The control unmodified Ad5-eGFP expressing eGFP in E1 region under the control of CMV promoter was prepared as previously described (17). The virus particle titers were detected by spectrophotometry at an absorbance (A) of $260 \mathrm{~nm}$.

Adenovirus transduction in vitro. A172, A549, U87 or U251 cells were seeded into a 24 -well plate at the density of $5 \times 10^{4}$ cells/well the day prior to the infection with Ad5-Hexon-SWDI or Ad5-eGFP at 5,000 vp/cell for A172 cells and 1,000 vp/ cell for the other 3 cell lines. Representative areas were photographed using the inverted fluorescence microscope at x200 magnification.

Statistical analysis. Statistical analysis for all experimental conditions was performed using the Statistical Package for the Social Sciences (SPSS) software (version 13; SPSS Inc., Chicago, IL, USA) by using one-way analysis of variance between groups (ANOVA/LSD).

\section{Results}

In vitro selection of phages. The level of CAR expression in some glioma cell lines, such as A172 cells, is low, which is consistent with limited adenoviral infection observed in our preliminary studies. For the identification of an alternative binding ligand to improve viral transduction of A172 cells, we performed panning with PhD-12 library, which consists of randomized linear 12-mer peptides. Phage clones that could specifically bind to A172 cells were isolated by 4 subtraction/ selection rounds. We used A172 for selections, and HEK 293 cells for subtractions. In each round, the bound phages were rescued and amplified in ER2738 for the following round of panning. After four subtraction/selection rounds, an aliquot of the phage pool was plated out, and plaques were picked for purification and sequencing. A total of 12 phage clones were chosen randomly from the final round of biopanning and subjected to DNA sequencing. The sequences of the displayed 

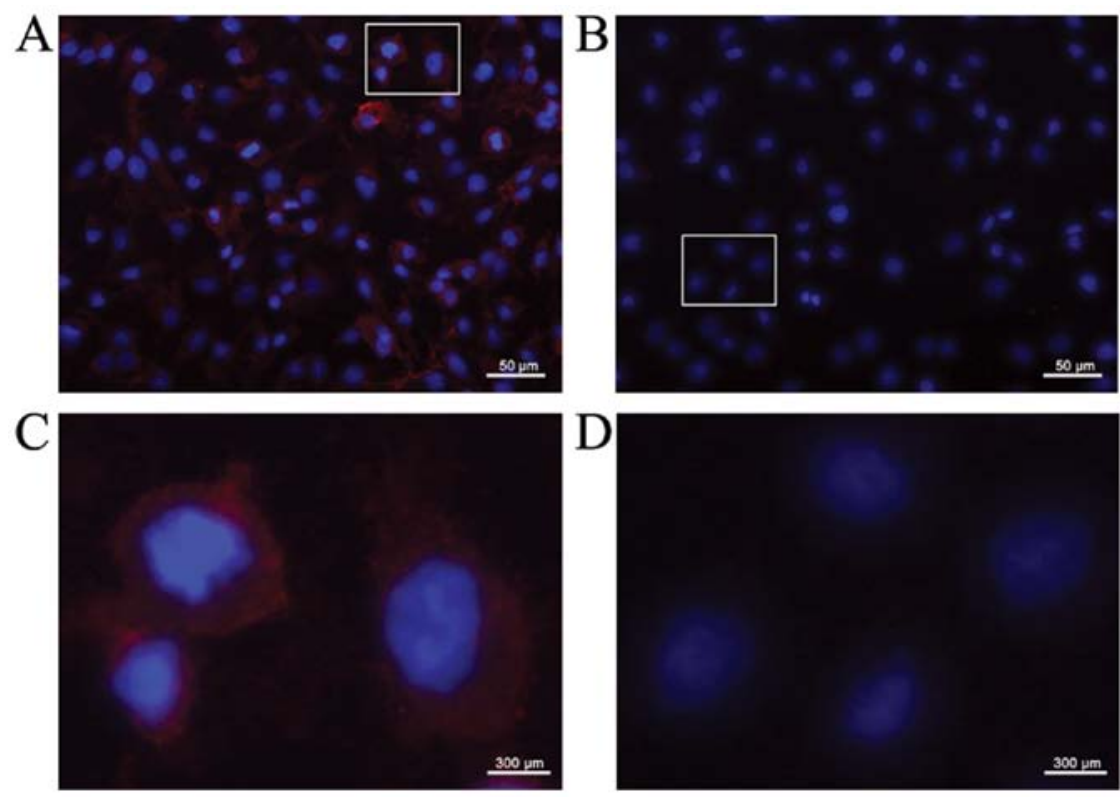

Figure 1. Detection of cell bound-phage by immunofluorescence staining. A172 cells incubated with phage SWDI (A) or the negative control, phage ASNL (B) were immunostained with anti-M13 phage antibody. C, insert in panel A; D, insert in panel B.

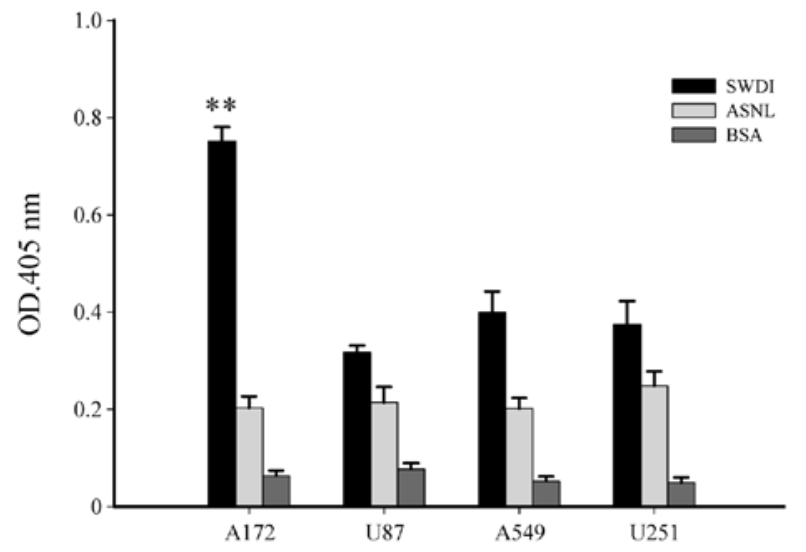

Figure 2. Specific binding of phage SWDI to A172 evaluated by phageELISA. U87, A549 and U251 were used as the control cells. The ASNL phage, the random phage from the phage libraries, was used as the control phage, and BSA protein as the mock control. The results represent the mean values \pm SEM (standard error of the mean) of two independent experiments analyzed in triplicate. ${ }^{* * *} \mathrm{P}<0.01$.

peptides are shown in Table I. The phage SWDI that appeared most frequently was selected as candidate.

Specific binding of phage SWDI to A172. Immunofluorescence staining with anti-M13 phage antibody was performed to observe the specific binding of phage SWDI to A172 cells. SWDI phages revealed by the red fluorescent foci were seen on the cell surface of A172 (Fig. 1A and C); however, no fluorescent signals were present when A172 cells were incubated with the negative control, phage ASNL, which was picked randomly from phage library (Fig. 1B and D).

To confirm the specific binding of phage SWDI to A172 cells, ELISA was performed using anti-M13 antibody. In addition to A172 cells, two other human glioblastoma cell lines, U87 and U251, and human lung carcinoma cell line A549 were used as the control cell lines. As shown in Fig. 2, the binding

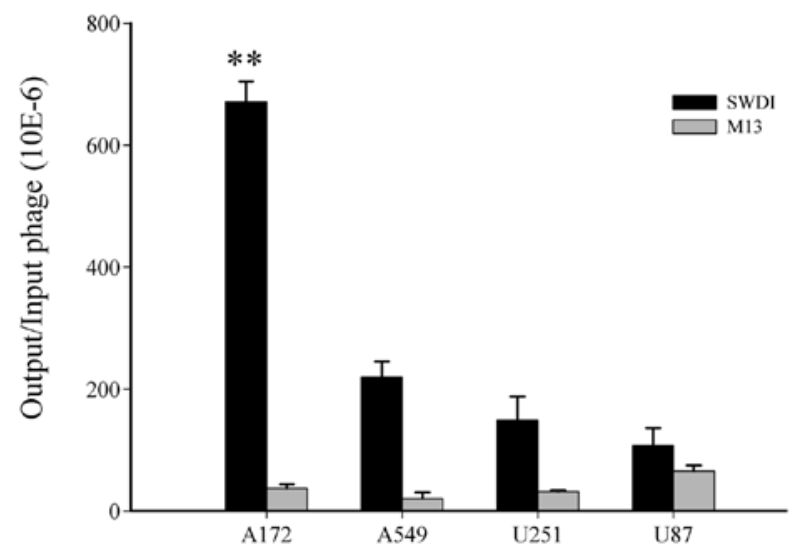

Figure 3. Binding efficiency of phage SWDI in different cell lines. Phage SWDI or the control wild-type M13 (both $5 \times 10^{9} \mathrm{pfu}$ ) was incubated with different cells $\left(1 \times 10^{6}\right.$ cells $)$ at $4^{\circ} \mathrm{C}$. After tittering, the phage binding efficiency was calculated as number of output phage/number of input phage. Data are presented as means \pm SEM (standard error of the mean) of triplicate experiments. ${ }^{* *} \mathrm{P}<0.01$.

activity of phage SWDI to A172 cells was 2.7-fold higher than that of phage ASNL to A172 (P<0.01). In U87, U251 and A549 cells, the binding activity of phage SWDI showed no significant difference when compared to that of ASNL.

Then, phage SWDI was incubated with A172 cells $\left(5 \times 10^{3}\right.$ pfu/cell) at $4^{\circ} \mathrm{C}$ to further evaluate the phage binding efficiency, which was calculated as number of output phage/number of input phage (Fig. 3). In A172 cells, the binding efficiency of phage SWDI was $\sim 13$-fold higher than that of the negative control wild-type M13 phage $(\mathrm{P}<0.01)$. There was no significant difference in binding efficiency between phage SWDI and M13 in other cell lines.

Generation of adenovirus with SWDI modified hexon. We utilized our previously constructed shuttle vector pRGHMAd5 (21), which contains a lacZ expression cassette in the hexon 


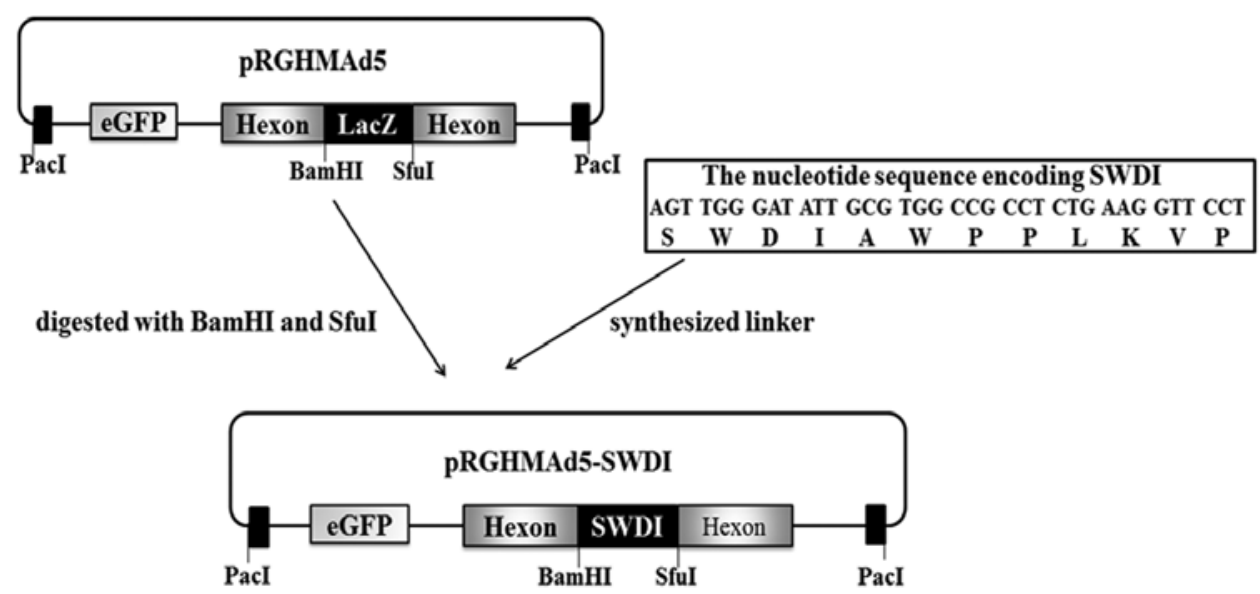

Figure 4. Diagram of the scheme used to construct the hexon-modified adenovirus vector. pRGHMAd5 virus vector, which contains a lacZ expression cassette in the hexon HVR5 region and an eGFP expression cassette driven by the CMV promoter in E1 region, was digested by BamHI and SfuI and ligated with oligonucleotides corresponding to SWDI, resulting in pRGHMAd5- SWDI.

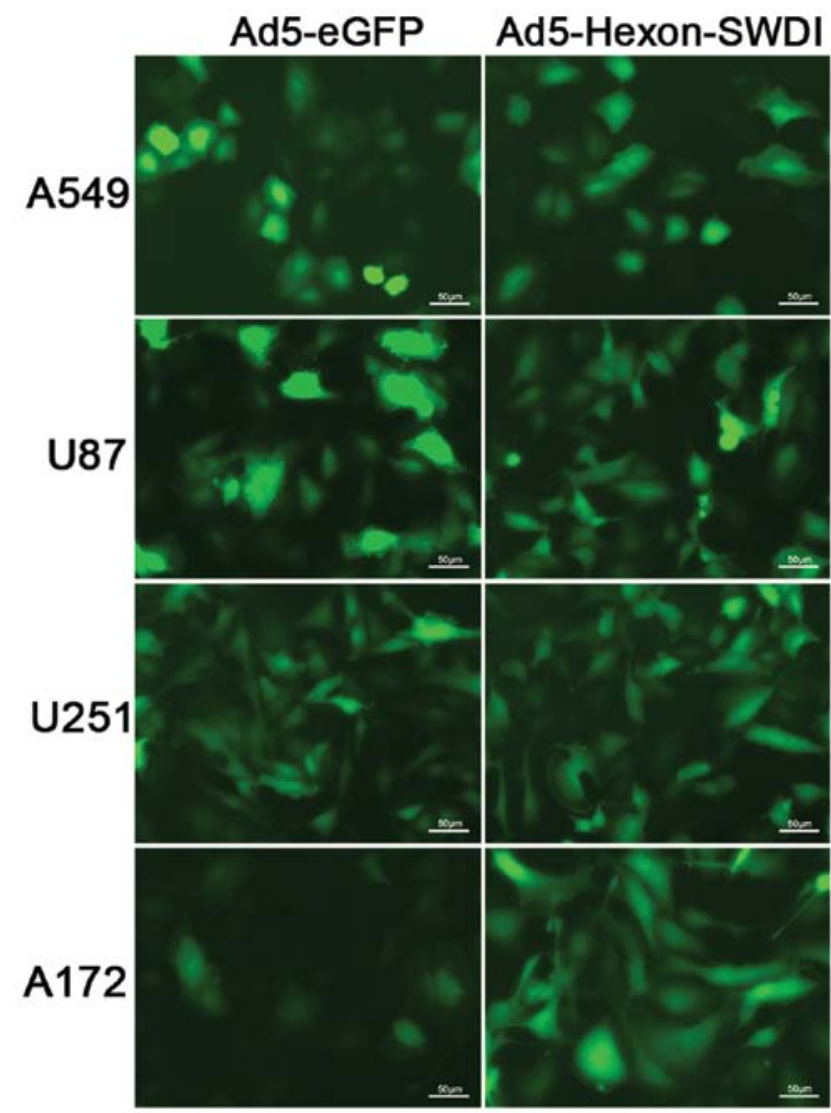

Figure 5. Comparison between Ad5-Hexon-SWDI and control virus Ad5-eGFP for transduction efficiency. A172 and control cell lines, A549, U87 and U251, were infected by Ad5-Hexon-SWDI or Ad5-eGFP. The representative images of eGFP expression for all indicated cells were captured under the fluorescent microscope $48 \mathrm{~h}$ post infection.

HVR5 region and an eGFP expression cassette driven by the CMV promoter in E1 region (Fig. 4), to incorporate peptide SWDI into the HVR5 of the Ad5 hexon. Peptide SWDI was ligated to the BamHI and SfuI digested pRGHMAd5. The obtained plasmid, termed pRGHMAd5-SWDI (Fig. 4), was confirmed by the restriction endonuclease analysis and DNA sequencing. Then, adenovirus with SWDI modified-hexon was successfully generated by transfecting PacI-linearized pRGHMAd5-SWDI into HEK 293 cells. The obtained virus was termed Ad5-Hexon-SWDI. The virus was produced with a titer of $2.8 \times 10^{11} \mathrm{vp} / \mathrm{ml}$ and the titer of the control virus Ad5-eGFP is $2.2 \times 10^{12} \mathrm{vp} / \mathrm{ml}$.

Transduction efficiency of adenovirus with SWDI-modified hexon. To examine the transduction efficiency of SWDImodified Ad, A172 and control cell lines including A549, U251 and U87 were infected with Ad5-Hexon-SWDI and control virus, Ad5-eGFP. Fig. 5 shows that the eGFP expression level in A172 infected with Ad5-Hexon-SWDI was higher than that in A172 infected with Ad5-eGFP. In each control cell line, no apparent difference in eGFP expression was observed between cells infected with Ad5-Hexon-SWDI and Ad5-eGFP. The results indicated that SWDI modification of hexon protein could improve the adenoviral transduction in CAR-deficient A172 cells specifically. GFP expression quantification by FACS revealed that GFP positivity ratio was higher in A172 treated by Ad5-Hexon-SWDI $(86.8 \pm 1.3 \%)$ than that in cells treated by Ad5-eGFP $(34.3 \pm 1.7 \%)(\mathrm{P}<0.01)$.

\section{Discussion}

In the present study, a new peptide SWDIAWPPLKVP, capable of selectively targeting a human glioblastoma cell line A172, was identified by direct biopanning of phage-display peptide libraries. The binding activity of SWDIAWPPLKVP peptide in A172 was more than 10-fold higher than that of the control phage. In addition, modification of hexon protein with this peptide could improve adenoviral infectivity in A172 cells.

Gene therapy is being studied in clinical trials for different types of cancer $(23,24)$. The features of Ad, such as high viral titer, high transduction efficiency for both proliferating and quiescent cells, large insertional capacity for foreign genes and high safety profile, make it well suited for cancer gene therapy (1). However, the progress of the application of Ad in 
cancer gene therapy is hindered by the lack of the expression of native adenovirus receptor on a variety of cancer types. To enable both efficient transduction of target cells and to avoid ectopic infection of non-target cells, strategies are needed to retarget the Ad vector to non-native cellular surface receptors.

A number of strategies have been developed, primarily based on the use of bispecific antibodies $(25,26)$, creation of fiber-chimeric Ad (13-16) and direct incorporation into viral capsid of peptide analogues specific for a tumor-associated receptor (17-21). The latter has been preferred for cancer cellspecific Ad retargeting, in which finding new cancer targeting peptides is crucial. On this path, phage display has been shown to be a powerful tool in identifying specific ligands on target cancer cells, such as glycine-serine-leucine (GSL) of breast carcinomas (27), asparagine-glycine-arginine (NGR) of tumor neovasculature $(28,29)$, and HEWSYLAPYPWF of colorectal cancer (30).

The fiber is the most commonly used capsid protein for retargeting Ad. Studies have shown that this location can be modified without interfering with capsid assembly (31). Several groups have successfully inserted cellular-specific targeting peptides into the HI loop of adenovirus fiber protein (17-19). Notably, in the present study, the Ad failed to propagate when peptide SWDIAWPPLKVP was inserted into the fiber HI-loop. Alternatively, we modified another capsid protein, hexon, within which the hypervariable regions (HVR) have solvent exposed loops, an ideal location for modification. Previous studies showed that genetic modification of HVR with integrinbinding RGD motif (32) had no effect on hexon structure or capsid stability but increased CAR-independent viral transduction. We took advantage of the adenoviral vector system for rapid modification of hexon protein, which was described in our previous study (21), and successfully generated a modified Ad with SWDI peptide inserted in hexon. The SWDI peptidemodified Ad was produced with decent titer, indicating that the viral assembly was not interfered by this modification. In addition, this peptide modification of Ad improved cell-specific adenoviral transduction.

In conclusion, these findings demonstrated that a peptide acquired through phage display can mediate cell-specific Ad retargeting when inserted into Ad hexon, suggesting an approach for targeting adenoviral infection to specific cancer cells. In the future, in vivo studies will be carried out to confirm the retargeting ability of the modified Ad in a glioblastoma animal model.

\section{Acknowledgements}

This study was supported by the Fundamental Research Funds for the Central Universities (GK201104004 and GK201301010), the Innovation Funds of Graduate Programs, Shaanxi Normal University (2011CXB006) and the National Natural Science Foundation of China (no. 81272543).

\section{References}

1. Breyer B, Jiang W, Cheng H, et al: Adenoviral vector-mediated gene transfer for human gene therapy. Curr Gene Ther 1: 149-162, 2001 .
2. Kim M, Zinn KRB, Barnett G, et al: The therapeutic efficacy of adenoviral vectors for cancer gene therapy is limited by a low level of primary adenovirus receptors on tumour cells. Eur J Cancer 38: 1917-1926, 2002.

3. Wickham TJ, Roelvink PW, Brough, et al: Adenovirus targeted to heparan-containing receptors increases its gene delivery efficiency to multiple cell types. Nat Biotechnol 14: 1570-1573, 1996.

4. Krasnykh VN, Mikheeva GV, Douglas JT, et al: Generation of recombinant adenovirus vectors with modified fibers for altering viral tropism. J Virol 70: 6839-6846, 1996.

5. Tomko RP, Xu R and Philipson L: HCAR and MCAR: the human and mouse cellular receptors for subgroup $C$ adenoviruses and group B coxsackieviruses. Proc Natl Acad Sci USA 94: 3352-3356, 1997.

6. Bergelson JM, Cunningham JA, Droguett G, et al: Isolation of a common receptor for coxsackie $B$ viruses and adenoviruses 2 and 5. Science 275: 1320-1323, 1997.

7. Bergelson JM, Krithivas A, Celi L, et al: The murine CAR homolog is a receptor for coxsackie B viruses and adenoviruses. J Virol 72: 415-419, 1998.

8. Roelvink PW, Lizonova A, Lee JG, et al: The coxsackievirusadenovirus receptor protein can function as a cellular attachment protein for adenovirus serotypes from subgroups A, C, D, E and F. J Virol 72: 7909-7915, 1998.

9. Wickham TJ, Matthias P, Cheresh DA, et al: Integrins $\alpha_{\mathrm{v}} \beta_{3}$ and $\alpha_{\mathrm{v}} \beta_{5}$ promote adenovirus internalization but not virus attachment. Cell 73: 309-319, 1993.

10. Nemerow GR, Pache L, Reddy V and Stewart PL: Insights into adenovirus host cell interactions from structural studies. Virology 384: 380-388, 2009.

11. Eto Y, Gao JQ, Sekiguchi F, et al: PEGylated adenovirus vectors containing RGD peptides on the tip of PEG show high transduction efficiency and antibody evasion ability. J Gene Med 7: 604-612, 2005.

12. Wortmann A, Vöhringer S, Engler T, et al: Fully detargeted polyethylene glycol-coated adenovirus vectors are potent genetic vaccines and escape from pre-existing anti-adenovirus antibodies. Mol Ther 16: 154-162, 2008.

13. Sakurai F, Mizuguchi H and Hayakawa T: Efficient gene transfer into human $\mathrm{CD} 34^{+}$cells by an adenovirus type 35 vector. Gene Ther 10: 1041-1048, 2003.

14. Wohlfahrt ME, Beard BC, Lieber A and Kiem HP: A capsidmodified, conditionally replicating oncolytic adenovirus vector expressing TRAIL leads to enhanced cancer cell killing in human glioblastoma models. Cancer Res 67: 8783-8790, 2007.

15. Havenga MJ, Lemckert AA, Grimbergen JM, et al: Improved adenovirus vectors for infection of cardiovascular tissues. J Virol 75: 3335-3342, 2001

16. Goossens PH, Havenga MJ, Pieterman E, et al: Infection efficiency of type 5 adenoviral vectors in synovial tissue can be enhanced with a type 16 fiber. Arthritis Rheum 44: 570-577, 2001.

17. Xia H, Anderson B, Mao Q and Davidson BL: Recombinant human adenovirus: targeting to the human transferrin receptor improves gene transfer to brain microcapillary endothelium. J Virol 74: 11359-11366, 2000.

18. Rittner K, Schreiber V, Erbs P and Lusky M: Targeting of adenovirus vectors carrying a tumor cell-specific peptide: in vitro and in vivo studies. Cancer Gene Ther 14: 509-518, 2007.

19. Jullienne B, Vigant F, Muth E, et al: Efficient delivery of angiostatin K1-5 into tumors following insertion of an NGR peptide into adenovirus capsid. Gene Ther 16: 1405-1415, 2009.

20. Liu S, Mao Q, Zhang W, et al: Genetically modified adenoviral vector with the protein transduction domain of Tat improves gene transfer to CAR-deficient cells. Biosci Rep 29: 103-109, 2009.

21. Di B, Mao Q, Zhao J, et al: A rapid generation of adenovirus vector with a genetic modification in hexon protein. J Biotechnol 157: 373-378, 2012.

22. Giordano RJ, Cardó-Vila M, Lahdenranta J, et al: Biopanning and rapid analysis of selective interactive ligands. Nat Med 7: 1249-1253, 2001

23. Alexander BL, Ali RR, Alton EW, et al: Progress and prospects: gene therapy clinical trials (part 1). Gene Ther 14: 1439-1447, 2007.

24. Aiuti A, Bachoud-Lévi AC, Blesch A, et al: Progress and prospects: gene therapy clinical trials (part 2). Gene Ther 14: 1555-1563, 2007. 
25. Volpers C, Thirion C, Biermann V, et al: Antibody-mediated targeting of an adenovirus vector modified to contain a synthetic immunoglobulin g-binding domain in the capsid. J Virol 77: 2093-2104, 2003.

26. Haisma HJ, Grill J, Curiel DT, et al: Targeting of adenoviral vectors through a bispecific single-chain antibody. Cancer Gene Ther 7: 901-904, 2000.

27. Arap W, Pasqualini R and Ruoslahti E: Cancer treatment by targeted drug delivery to tumor vasculature in a mouse model Science 279: 377-380, 1998.

28. Curnis F, Arrigoni G, Sacchi A, et al: Differential binding of drugs containing the NGR motif to CD13 isoforms in tumor vessels, epithelia, and myeloid cells. Cancer Res 62: 867-874, 2002.
29. Pasqualini R, Koivunen E, Kain R, et al: Aminopeptidase $\mathrm{N}$ is a receptor for tumor-homing peptides and a target for inhibiting angiogenesis. Cancer Res 60: 722-727, 2000.

30. Rasmussen UB, Schreiber V, Schultz H, et al: Tumor celltargeting by phage-displayed peptides. Cancer Gene Ther 9 : 606-612, 2002.

31. Belousova N, Krendelchtchikova V, Curiel DT and Krasnykh V: Modulation of adenovirus vector tropism via incorporation of polypeptide ligands into the fiber protein. J Virol 76: 8621-8631, 2002.

32. Vigne E, Mahfouz I, Dedieu JF, et al: RGD inclusion in the hexon monomer provides adenovirus type 5-based vectors with a fiber knob-independent pathway for infection. J Virol 73: 5156-5161, 1999. 